\title{
The effects of Mobilization and Stimulation of Neuromuscular Tissue on the hemiplegic upper limb: a case report
}

\author{
DOI: https://doi.org/10.5114/pq.2019.83055 \\ Dimitrios Athanasiadis ${ }^{1}$, Stylianos Protopsaltis ${ }^{2}$, Eleftherios Stefas ${ }^{3}$ \\ ${ }^{1}$ Physiotherapy Practice Dimitrios Athanasiadis, Kavala, Greece \\ ${ }^{2}$ latriki Askisi Rehabilitation Clinic, Athens, Greece \\ ${ }^{3}$ Evexia Rehabilitation Clinic, Thessaloniki, Greece
}

\section{Abstract}

Introduction. Mobilization and Stimulation of Neuromuscular Tissue (MaSoNT) is a newly-invented technique that aims to trigger functional recovery in stroke patients. This is the first study that applies an intervention with MaSoNT in a patient's hemiplegic hand. The purpose was to investigate the effects of a MaSoNT intervention and to prove the safety of the technique. Methods. A case study is provided. The patient was a male stroke survivor, aged 71 , who underwent a cerebrovascular accident a week before the intervention. He presented zero voluntary movement in the hemiplegic arm and some sensory impairment. The patient had had neither pain nor spasticity before the intervention. The intervention was standard upper limb therapy performed twice per week and MaSoNT application for 15 minutes, 5 times per week. The total duration of the intervention was 3 weeks. The following measurement tools were used: Modified Ashworth Scale, Motricity Index Arm Score, Motor Assessment Scale items 6, 7, and 8, Thumb Localization Test, Nottingham Sensory Assessment.

Results. The patient demonstrated both motor and sensory improvements by the end of the experiment. Pain and spasticity remained absent.

Conclusions. The study possibly proves that MaSoNT is a safe technique to apply in a hemiplegic hand. Moreover, it could lead to functional recovery, although further research is mandated.

Key words: MaSoNT, functional recovery, proof of safety, stroke

\section{Introduction}

Every year, there are approximately 100,000 stroke incidents in the UK, with the majority of those patients leaving hospital with disability [1]. The upper limb is most commonly affected by permanent disability [2]; merely less than 2 out of 10 stroke patients experience full recovery of function in the upper extremity [3]. Overall, 6 out of 10 stroke survivors could not manage to recover some dexterity of the hemiplegic hand even after a 6-month intervention [4]. Hence, even though many 'hands-on' techniques are applied in order to trigger functional recovery to the upper limb [5], a vast majority of them failed to establish a standard of usefulness in that regard [6]; there is a necessity for more evidence-based practice as well as a better reasoning for their application [7].

Mobilization and Stimulation of Neuromuscular Tissue (MaSoNT) is a newly-invented sensory facilitatory technique for a hemiplegic upper limb where the therapist offers somatosensory stimuli aiming to trigger functional recovery through cortical reorganization [8]. To further analyse the rationale underpinning MaSoNT in neurorehabilitation, one must note that during the post-stroke stages, there is a sequence of events essential for recovery, and cortical reorganization is its neural basis [8]. Cortical reorganization happens anyway after injury but it can be maladaptive or non-effective [8]. Therefore, the therapist applies sensory facilitatory techniques in order to trigger and guide the reorganizational changes of the cortex in favour of functional recovery [8].

In past years, it was shown that interventions focusing on somatosensory stimulation - similar to MaSoNT - could cause cortical reorganization in stroke survivors and thus trigger the initiation of this sequence of events that would finally lead to recovery [8]. The safety of the technique has been studied and supported in theory [8], and an exploratory study was conducted in a small number of stroke patients [9]. This case study primarily aims to prove the safety of MaSoNT. Its secondary purpose is to report MaSoNT effects regarding pain, spasticity, and recovery.

\section{Subject and methods}

\section{Participant}

As the patient was the first one to receive MaSoNT in a monitored and regular manner, the sample had to be of convenience. The final patient was a 71-year-old male stroke survivor classified to have a lacunar circulation ischemic stroke syndrome [10]. The areas of infarct were the pons and the basal ganglia. The dominant side was the hemiplegic, right one. The patient was recruited on the basis of being more suitable for the technique according to evidence [8]. The patient received MaSoNT one week after the cerebrovascular accident, which was his second. The first stroke was mild and ischemic, infarcted the left pons, occurred 2 years before the second one and presented neither motor nor sensory deficits. With the second stroke, which was also ischemic, the patient presented dysarthria, positive (+) Babinski sign on the hemiplegic side, and no aphasia. His body mass index pointed at overweight. Moreover, the patient showed a history of hyperlipidaemia, hypertension, diabetes

Correspondence address: Dimitrios Athanasiadis, MSc, PT, Physiotherapy Practice Dimitrios Athanasiadis, 8B, Mitropoleos street, Kavala, 65403, Greece. Professor in the Department of Physiotherapy and Occupational Therapy at the Institute of Vocational Training (I.E.K.) of Kavala, Greece 
Table 1. The patient's baseline features

\begin{tabular}{|l|c|}
\hline Gender & male \\
\hline Age (years) & 71 \\
\hline Days since stroke & 7 \\
\hline Hemiplegic side & right \\
\hline Dominant side & right \\
\hline Pain / No pain & no pain \\
\hline National Institutes of Health Stroke Scale & 14 \\
\hline Functional Independence Measure & 33 \\
\hline Mini-Mental State Examination & 25 \\
\hline Modified Ashworth Scale & 0 \\
\hline Motricity Index Arm Score & 0 \\
\hline Motor Assessment Scale items 6, 7, and 8 & 0 \\
\hline Thumb Localization Test & 6 \\
\hline & $\begin{array}{c}\text { tactile sensation: } 1 \\
\text { kinaesthetic } \\
\text { sensations: } 1 \\
\text { stereognosis: } 2\end{array}$ \\
\hline Nottingham Sensory Assessment & \\
\hline
\end{tabular}

mellitus type 2, chronic kidney disease, and deep vein thrombosis at the superficial femoral vein and the popliteal vein. The pharmaceutical treatment included: Actrapid, Lantus, Ivor 2500 IU, TBS Salospir, omeprazole, Amlopen, Coaprovel, TBS Atorstat 20, TBS Hytrin. Furthermore, the subject had received intravenous injections of recombinant tissue plasminogen activator (tPA) when in acute phase. The shoulder abduction and finger extension (SAFE) score showed no voluntary motor control within 72 hours after the cerebrovascular accident, which shows low predictability of regaining dexterity at 6 months [11]. The physiotherapy intervention was the first one that the patient received after the stroke. The patient's clinical features are presented in Table 1.

\section{Study design}

As evidenced elsewhere [8], the intervention offered was standard upper limb therapy applied twice per week for a quarter of an hour, along with MaSoNT. The standard upper limb therapy included passive/active movement exercises and static/dynamic stretching. No electrically generated stimuli were offered (device-free). MaSoNT was applied for 15 minutes, 5 times per week. The experiment lasted 3 weeks. The positioning of the hemiplegic upper limb was offered twice a day for 30 minutes [12]. The study design abides by the CARE guidelines [13] for case reports.

\section{Measurement tools}

In order to assess pain, visual analogue scale was not preferred owing to the criticism on its use in stroke survivors [14]. Pain dichotomous evaluation was used instead (pain versus no pain) as inspired by other studies on stroke survivors [15]. Pain was assessed both at rest and at the moment of the hemiplegic arm movement. To assess spasticity at the shoulder, elbow, and wrist, the Modified Ashworth Scale [16] was used; its psychometric application among stroke patients is supported [17-22] but it also has some limitations [23-25]. Moreover, to assess motor function, the
Motricity Index [26] was used, which is widely evidence-based [27-31], along with items 6, 7, and 8 of the Motor Assessment Scale [32], again supported by evidence [33-36]. Lastly, the Thumb Localization Test was utilized to assess proprioception $[37,38]$, and the Nottingham Sensory Assessment to determine the somatosensory effects $[39,40]$. The independent assessor was a physiotherapist who was blinded to the intervention. However, there was no blinding for the patient.

\section{MaSoNT application procedure}

MaSoNT is a concept that, at its basic form, involves the application of a transverse stretch across the muscle belly, either in the upper or in the lower limb. Moreover, there should be a progression in the way to apply the MaSoNT basic technique, depending on the attendance and functional level of the patient [41].

A basic rule for MaSoNT - which also applies to other forms of therapeutic approaches - is that the patient must be deprived of any other form of external stimuli [41]. It is necessary to keep the patient focused on the application and on what the therapist is trying to achieve by stimulating the limb. Also, as a general rule, MaSoNT techniques are recommended to be used in combination with one another, in accordance with the patient's condition and treatment goals [41].

In the presented study, the patient received 4 different MaSoNT techniques: the basic facilitatory technique for the upper limb, twisting of the upper limb, finger swiping, and pinching [41]. For the basic facilitatory technique, the upper limb was lifted by the therapist with specific handling and brought towards the patient's field of view in order to gain his full attention. The position acquired through the therapists' handling was: shoulder in flexion, adduction, mid-rotation, elbow in mid-flexion, and forearm in pronation. The wrist, along with the fingers, was free of handling, and thus placed relaxingly by gravity force in flexion. The application dosage was 4 times per minute with 15-second interval.

Seconds before applying the basic facilitatory technique, the therapist instructed the patient to focus on the hand and the contraction that was going to occur. The therapist targeted the muscle belly and applied a transverse stretch. A brisk contraction was seen as a result of this application. If a contraction was not elicited once applied over a particular spot, an additional application was offered in another spot that would trigger it. If even an additional application could not elicit a contraction, no other effort was attempted because of safety reasons for the biomechanical infrastructure of the neuromusculature.

The application was offered at 4 different spots and included several areas of the forearm dorsal surface, aiming to trigger the extensors muscle group. One application spot also included the brachioradialis muscle belly area, with the purpose to trigger this particular muscle, which could elicit contraction with regard to the elbow. Such an application spot could not be detected for the triceps brachii insertion close to the olecranon.

For the twisting technique, in the starting position, the therapist supported the forearm with the left hand in a relaxed position and placed the thumb of the right hand over the muscle belly. Afterwards, the therapist applied a continuing transverse stretch on the muscle belly with the right hand and supinated the patient's hand. The therapist held this position for a longer time period, about 30 seconds, and then tried it again after a while, in an area located lower or higher than that of the initial application. Tapping and some 
light joint compression was offered during the application intervals.

In the finger swiping technique, the therapist brought the limb to a relaxed position. At first, the therapist closed 2 of their fingers (or 3 , if needed), the index and the middle finger, and aimed diagonally to swipe over the muscle belly of the desired area of facilitation. The therapist hit abruptly and in short intervals along the desired area that needed to be facilitated, and a brisk contraction was apparent.

When applying the pinching technique, the therapist supported the patient's hand and supinated it. With the patient's palmar surface exposed and supported, the therapist brought the hand close to the visual field of the patient and stimulated several areas of the palmar surface by slightly pinching them. The therapist asked the patient to inform about the quality of the sensation - if there was any - in order to make him focus on the sensation and practise on it. The therapist pinched several spots on the palmar surface and the fingers for about 5 minutes in total.

Additionally, it must be mentioned that vulnerable application spots of the radial nerve-were avoided in all applications.

\section{Ethical approval}

The research related to human use has been complied with all the relevant national regulations and institutional policies, has followed the tenets of the Declaration of Helsinki, and has been approved by the authors' institutional review board.

\section{Informed consent}

Informed consent has been obtained from the individual included in this study.

\section{Results}

The outcomes of the intervention are summarized in Table 2. Regarding pain, no increase was noticed in week 3 either at rest or at movement in the whole upper limb (including the shoulder). Spasticity was not raised. The Motricity Index showed an increase of $47 \%$, while the Motor Assessment Scale revealed a $28 \%$ improvement compared with baseline. Lastly, the Thumb Localization Test presented a 25\% increase, and the Nottingham Sensory Assessment demonstrated a $33 \%$ increase in tactile sensation and $25 \%$ in kinaesthesia. No harmful effect was present with regards to stereognosis, which was unaltered.

Early during the experiment, while the thumb, index, and middle finger presented voluntary movement, no such effect was shown in the ring or little finger. Consequently, the ther- apist started aiming more laterally and distantly to the elbow in order to trigger the respective neuromusculature. Small movements of the index and middle finger were apparent within week 1.

Moreover, in the middle of week 2 of the experiment, a taut band appeared medially at the surface of application on the forearm. No tenderness or pain pattern was apparent under palpation, under ischemic compression, or in calm. It was speculated to be a latent (silent) trigger point [42] and it was never used as an application spot again. This clinical sign disappeared by the end of week 3 .

Contractures were not apparent. Evidence indicates that the earliest contractures can be apparent 2 months after stroke [43]. Moreover, given that early functional recovery was achieved at week 3 , the patient was not likely to present any contractures in the future [44].

\section{Discussion}

This is the first clinical study on MaSoNT and the effects of its use in a hemiplegic arm. MaSoNT belongs to a group of sensory facilitatory techniques that can be applied in everyday clinical practice in order to assist functional recovery. The group of techniques could be named 'zero-to-one' techniques as they aim to improve function of a flaccid hemiplegic hand from no voluntary movement (zero condition) to at least some movement (one condition) upon which another therapeutic approach can build on and improve to an even better condition. The study showed that the effect MaSoNT had on the patient was positive, even though the SAFE score predicted otherwise [11].

Previous studies implemented similar interventions of somatosensory stimulation. Sensorimotor training improved the functional recovery of 2 chronic stroke survivors in a 2-week intervention with neural reorganization being induced [45]. Moreover, a program of stretching, range of motion exercises, and soft tissue mobilization techniques offered to 5 chronic stroke patients in a 3-week intervention managed to provide functional improvement along with cortical reorganization [46]. Noteworthy, the current study is the first to apply such a sensorimotor intervention in a hemiplegic hand so early after stroke.

Regarding cortical reorganization measurements, the rationale of MaSoNT use is to offer functional recovery by eliciting cortical reorganization [8]. As no imaging scanning device was implemented in the study owing to financial reasons, no information on the effect of the intervention on cortical reorganization could be granted. It could be speculated that neuroplastic reorganizational alterations did occur in the pa-

Table 2. Scores at baseline and week 3

\begin{tabular}{|l|c|c|}
\hline Outcome measures & Baseline & Week 3 \\
\hline Pain / No pain at the whole upper limb & no pain & 0 \\
\hline Modified Ashworth Scale & 0 & noin \\
\hline Motricity Index Arm Score & 0 & total: 47 \\
\hline Motor Assessment Scale items 6, 7, and 8 & 0 & total: 5 \\
Thumb Localization Test & 6 & (item 6: 14; item 7: 2; item 8: 2)
\end{tabular}


tient's brain; otherwise, no functional recovery would be seen at all [47]. However, whether these cortical reorganizational changes and their extent are to be attributed to the intervention is questionable as some physiological neuroplastic changes would occur naturally [48]. Unknown mechanisms can trigger motor recovery through cortical reorganization when a sensorimotor technique is applied [49]. Evidence strongly indicates that therapeutic interventions can enhance functional recovery through cortical reorganization in stroke patients [8]. Notably, passive movement alone is able to trigger changes in cortical representation and excitability among healthy individuals [8].

Apart from the effects, the study can support the safety of the technique. Pain, both at rest and in movement, remained absent before and after the intervention. Certainly, this does not imply that pain will not be apparent for the patient in the future as this phenomenon is highly prevalent 6 months after stroke [50]. Additionally, there was no negative effect regarding the development of spasticity. Again, spasticity may appear as early as 2 weeks after stroke [51] and its prevalence increases at 3 [52] and 6 [53] weeks after stroke. However, given the low degree of motor and sensory deficit, as well as the absence of spasticity at this early stage, one can expect that the patient probably will not be seriously affected by spasticity $[51,53,54]$. Thus, some proof of the MaSoNT intervention safety can be granted by the current study.

\section{Limitations}

More assessment scales on functional recovery could have been included but the study did not primarily aim to it. Even with more such scales, in a convenience sample such as the one recruited, no spherical generalized conclusion could be reached. That was the second limitation of the study. Lastly, no blinding of the patients was implemented. This risk of bias diminishes the credibility of the results but when studying alternative innovative interventions, full blinding becomes almost impossible [55].

\section{Conclusions}

This study was the first one to offer MaSoNT early in a stroke patient's upper limb. The major conclusion is that MaSoNT possibly cannot cause any harmful effects on the recovery of the hemiplegic hand. Additionally, it might bring about motor and sensory improvement. Hence, it could be recommended in combination with the conventional treatment approach. Future research with a larger number of subjects is needed to validate the duration and doses and generalize the efficacy of the intervention in a greater stroke population.

\section{Acknowledgements}

We would like to acknowledge the Evexia Rehabilitation Clinic for permitting the conduction of the trial. Also, we thank the physiotherapist who was the independent assessor in the study.

\section{Disclosure statement}

No author has any financial interest or received any financial benefit from this research.

\section{Conflict of interest}

The authors state no conflict of interest.

\section{References}

1. State of the nation: stroke statistic. Stroke Association; 2017. Available from: https://www.stroke.org.uk/sites/ default/files/state_of_the_nation_2017_final_1.pdf.

2. Broeks JG, Lankhorst GJ, Rumping K, Prevo AJH. The long-term outcome of arm function after stroke: results of a follow-up study. Disabil Rehabil. 1999;21(8):357364; doi: 10.1080/096382899297459.

3. Sidaway M, Głowacka-Popkiewicz J, Krawczyk M, Waraksa T. Early upper limb physiotherapy in stroke patients. Questions without answers. Adv Rehabil. 2017;31(1): 37-47; doi: 10.1515/rehab-2015-0060.

4. Kwakkel G, Kollen BJ, van der Grond J, Prevo AJ. Probability of regaining dexterity in the flaccid upper limb: impact of severity of paresis and time since onset in acute stroke. Stroke. 2003;34(9):2181-2186; doi: 10.1161/01. STR.0000087172.16305.CD.

5. Jackson J. Specific treatment techniques. In: Stokes M, Stack E (eds.), Physical management for neurological conditions, $3^{\text {rd }}$ ed. Edinburgh: Elsevier; 2011; 243-266.

6. Langhorne P, Coupar F, Pollock A. Motor recovery after stroke: a systematic review. Lancet Neurol. 2009;8(8): 741-754; doi: 10.1016/S1474-4422(09)70150-4.

7. Langhorne P, Bernhardt J, Kwakkel G. Stroke rehabilitation. Lancet. 2011;377(9778):1693-1702; doi: 10.1016/ S0140-6736(11)60325-5.

8. Athanasiadis D. Physiology underpinning Mobilization and Stimulation of Neuromuscular Tissue (MaSoNT): a review. Master's thesis. Keele: Keele University; 2015. Available from: https://www.researchgate.net/profile/ Dimitrios_Athanasiadis2/publication/303288344_ Physiology_underpinning_Mobilization_and_Stimulation_of_Neuromuscular_Tissue_MaSoNT_a_review/ links/573b28a908ae9f741b2d78f0/Physiology-underpinning-Mobilization-and-Stimulation-of-Neuromuscular-Tissue-MaSoNT-a-review.pdf?origin=publication detail.

9. Athanasiadis D, Dionyssiotis Y, Papathanasiou J, Stefas E. Mobilization and Stimulation of Neuromuscular Tissue (MASONT) for stroke survivors. Folia Med. 2018; 60(1):95-99; doi: 10.1515/folmed-2017-0074.

10. Bamford J, Sandercock P, Dennis M, Burn J, Warlow C. Classification and natural history of clinically identifiable subtypes of cerebral infarction. Lancet. 1991;337(8756): 1521-1526; doi: 10.1016/0140-6736(91)93206-O.

11. Nijland $R H$, van Wegen EE, Harmeling-van der Wel BC, Kwakkel G, EPOS Investigators. Presence of finger extension and shoulder abduction within 72 hours after stroke predicts functional recovery: early prediction of functional outcome after stroke: the EPOS cohort study. Stroke. 2010;41(4):745-750; doi: 10.1161/STROKEAHA. 109.572065.

12. Ada L, Goddard E, McCully J, Stavrinos T, Bampton J. Thirty minutes of positioning reduces the development of shoulder external rotation contracture after stroke: a randomized controlled trial. Arch Phys Med Rehabil. 2005;86(2):230-234; doi: 10.1016/j.apmr.2004.02.031.

13. Gagnier JJ, Kienle G, Altman DG, Moher D, Sox H, Riley D, et al. The CARE guidelines: consensus-based clinical case reporting guideline development. Glob Adv Health Med. 2013;2(5):38-43; doi: 10.7453/gahmj.2013.008.

14. Price CIM, Curless RH, Rodgers H. Can stroke patients use visual analogue scales? Stroke. 1999;30(7):13571361; doi: 10.1161/01.STR.30.7.1357.

15. Pandyan AD, Cameron M, Powell J, Stott DJ, Granat MH. Contractures in the post-stroke wrist: a pilot study of its 
time course of development and its association with upper limb recovery. Clinl Rehabil. 2003;17(1):88-95; doi: 10.1191/0269215503cr587oa.

16. Bohannon RW, Smith MB. Interrater reliability of a modified Ashworth scale of muscle spasticity. Phys Ther. 1987;67(2):206-207; doi: 10.1093/ptj/67.2.206.

17. Allison SC, Abraham LD, Petersen CL. Reliability of the Modified Ashworth Scale in the assessment of plantarflexor muscle spasticity in patients with traumatic brain injury. Int J Rehabil Res. 1996;19(1):67-78; doi: 10.1097/00004356-199603000-00007.

18. Lin FM, Sabbahi M. Correlation of spasticity with hyperactive stretch reflexes and motor dysfunction in hemiplegia. Arch Phys Med Rehabil. 1999;80(5):526-530; doi: 10.1016/S0003-9993(99)90193-4.

19. Gregson JM, Leathley MJ, Moore AP, Smith TL, Sharma AK, Watkins CL. Reliability of measurements of muscle tone and muscle power in stroke patients. Age Ageing. 2000;29(3):223-228.

20. Blackburn M, van Vliet $P$, Mockett SP. Reliability of measurements obtained with the modified Ashworth scale in the lower extremities of people with stroke. Phys Ther. 2002;82(1):25-34; doi: 10.1093/ptj/82.1.25.

21. Brashear A, Zafonte R, Corcoran M, Galvez-Jimenez N, Gracies JM, Gordon MF, et al. Inter- and intrarater reliability of the Ashworth Scale and the Disability Assessment Scale in patients with upper-limb poststroke spasticity. Arch Phys Med Rehabil. 2002;83(10):1349-1354; doi: 10.1053/apmr.2002.35474.

22. Kaya T, Karatepe AG, Gunaydin R, Koc A, Ercan UA. Inter-rater reliability of the Modified Ashworth Scale and modified Modified Ashworth Scale in assessing poststroke elbow flexor spasticity. Int J Rehabil Res. 2011;34(1): 59-64; doi: 10.1097/MRR.0b013e32833d6cdf.

23. Pandyan AD, Johnson GR, Price CIM, Curless $R H$, Barnes MP, Rodgers H. A review of the properties and limitations of the Ashworth and modified Ashworth Scales as measures of spasticity. Clin Rehabil. 1999;13(5):373383; doi: 10.1191/026921599677595404.

24. Kamper DG, Schmit BD, Rymer WZ. Effect of muscle biomechanics on the quantification of spasticity. Ann Biomed Eng. 2001;29(12):1122-1134; doi: 10.1114/1. 1424918.

25. Salter K, Jutai JW, Teasell R, Foley NC, Bitensky J. Issues for selection of outcome measures in stroke rehabilitation: ICF Body Functions. Disabil Rehabil. 2005;27(4):191207; doi: 10.1080/09638280400008537.

26. Collin C, Wade DT. Assessing motor impairment after stroke: a pilot reliability study. J Neurol Neurosurg Psychiatry. 1990;53(7):576-579; doi:10.1136/jnnp.53.7.576.

27. Kopp B, Kunkel A, Flor H, Platz T, Rose U, Mauritz KH, et al. The Arm Motor Ability Test: reliability, validity, and sensitivity to change of an instrument for assessing disabilities in activities of daily living. Arch Phys Med Rehabil. 1997; 78(6):615-620; doi: 10.1016/S0003-9993(97)90427-5.

28. Hsieh CL, Hsueh IP, Chiang FM, Lin PH. Inter-rater reliability and validity of the action research arm test in stroke patients. Age Ageing. 1998;27(2):107-113; doi: 10.1093/ageing/27.2.107.

29. Bohannon RW. Motricity index scores are valid indicators of paretic upper extremity strength following stroke. J Phys Ther Sci. 1999;11(2):59-61; doi: 10.1589/jpts.11.59.

30. Safaz I, Yilmaz B, Yaşar E, Alaca R. Brunnstrom recovery stage and motricity index for the evaluation of upper extremity in stroke: analysis for correlation and responsiveness. Int J Rehabil Res. 2009;32(3):228-231;
31. Kumar P. Reliability of motricity index strength assessments for upper extremity in post stroke hemiparesis a correlation study. 2015. Available from: https://clinicaltrials.gov/ct2/show/NCT02353520.

32. Carr JH, Shepherd RB, Nordholm L, Lynne D. Investigation of a new motor assessment scale for stroke patients. Phys Ther. 1985;65(2):175-180.

33. Poole JL, Whitney SL. Motor assessment scale for stroke patients: concurrent validity and interrater reliability. Arch Phys Med Rehabil. 1988;69(3 Pt 1):195-197.

34. Malouin F, Pichard L, Bonneau C, Durand A, Corriveau D. Evaluating motor recovery early after stroke: comparison of the Fugl-Meyer Assessment and the Motor Assessment Scale. Arch Phys Med Rehabil. 1994;75(11):12061212; doi: 10.1016/0003-9993(94)90006-X.

35. Lannin NA. Reliability, validity and factor structure of the upper limb subscale of the Motor Assessment Scale (UL-MAS) in adults following stroke. Disabil Rehabil. 2004; 26(2):109-116; doi: 10.1080/0963828032000157970.

36. English CK, Hillier SL, Stiller K, Warden-Flood A. The sensitivity of three commonly used outcome measures to detect change amongst patients receiving inpatient rehabilitation following stroke. Clin Rehabil. 2006;20(1): 52-55; doi: 10.1191/0269215506cr877oa.

37. Hirayama K, Fukutake T, Kawamura M. 'Thumb localizing test' for detecting a lesion in the posterior columnmedial lemniscal system. J Neurol Sci. 1999;167(1):4549; doi: 10.1016/S0022-510X(99)00136-7.

38. Welmer AK, von Arbin M, Murray V, Holmqvist LW, Sommerfeld DK. Determinants of mobility and self-care in older people with stroke: importance of somatosensory and perceptual functions. Phys Ther. 2007;87(12):16331641; doi: 10.2522/ptj.20060349.

39. Lincoln NB, Jackson JM, Adams SA. Reliability and revision of the Nottingham Sensory Assessment for stroke patients. Physiotherapy. 1998;84(8):358-365; doi: 10.1016/ S0031-9406(05)61454-X.

40. Stolk-Hornsveld F, Crow JL, Hendriks EP, van der Baan R, Harmeling-van der Wel BC. The Erasmus MC modifications to the (revised) Nottingham Sensory Assessment: a reliable somatosensory assessment measure for patients with intracranial disorders. Clin Rehabil. 2006;20(2): 160-172; doi: 10.1191/0269215506cr932oa.

41. Athanasiadis $D$ (ed.). Mobilization and Stimulation of Neuromuscular Tissue (MaSoNT): a concept for stroke survivors. Beau Bassin: Lambert Academic Publishing; 2018.

42. Dommerholt J, Fernández-de-las-Peñas C (eds.). Trigger point dry needling. An evidenced and clinical-based approach. Oxford: Churchill Livingstone; 2013.

43. O'Dwyer NJ, Ada L, Neilson PD. Spasticity and muscle contracture following stroke. Brain. 1996;119(Pt 5):17371749; doi: 10.1093/brain/119.5.1737.

44. Pandyan AD, Cameron M, Powell J, Stott DJ, Granat $\mathrm{MH}$. Contractures in the post-stroke wrist: a pilot study of its time course of development and its association with upper limb recovery. Clin Rehabil. 2003;17(1):8895; doi: 10.1191/0269215503cr587oa.

45. Borstad AL, Bird T, Choi S, Goodman L, Schmalbrock P, Nichols-Larsen DS. Sensorimotor training induced neural reorganization after stroke: a case series. J Neurol Phys Ther. 2013;37(1):27-36; doi: 10.1097/NPT.0b013e3182 83de0d.

46. Nelles G, Jentzen W, Jueptner M, Müller S, Diener HC. Arm training induced brain plasticity in stroke studied 
with serial positron emission tomography. Neuroimage. 2001;13(6 Pt 1):1146-1154; doi: 10.1006/nimg.2001. 0757.

47. Nudo RJ. Neural bases of recovery after brain injury. J Commun Disord. 2011;44(5):515-520; doi: 10.1016/j. jcomdis.2011.04.004.

48. Shumway-Cook A, Woollacott MH (eds.). Motor control: translating research into clinical practice, $4^{\text {th }}$ ed. Philadelphia: Wolters Kluwer Health, Lippincott Williams \& Wilkins; 2012.

49. Flor H, Diers M. Sensorimotor training and cortical reorganization. NeuroRehabilitation. 2009;25(1):19-27; doi: 10.3233/NRE-2009-0496.

50. Hansen AP, Marcussen NS, Klit H, Andersen G, Finnerup NB, Jensen TS. Pain following stroke: a prospective study. Eur J Pain. 2012;16(8):1128-1136; doi: 10.1002/ j.1532-2149.2012.00123.x.

51. Wissel J, Schelosky LD, Scott J, Christe W, Faiss JH, Mueller J. Early development of spasticity following stroke: a prospective, observational trial. J Neurol. 2010;257(7): 1067-1072; doi: 10.1007/s00415-010-5463-1.

52. Sommerfeld DK, Eek EUB, Svensson AK, Holmqvist LW, von Arbin $\mathrm{MH}$. Spasticity after stroke: its occurrence and association with motor impairments and activity limitations. Stroke. 2004;35(1):134-139; doi: 10.1161/01. STR.0000105386.05173.5E

53. Urban PP, Wolf T, Uebele M, Marx JJ, Vogt T, Stoeter P, et al. Occurrence and clinical predictors of spasticity after ischemic stroke. Stroke. 2010;41(9):2016-2020; doi: 10.1161/STROKEAHA.110.581991.

54. Sommerfeld DK, Gripenstedt U, Welmer AK. Spasticity after stroke: an overview of prevalence, test instruments, and treatments. Am J Phys Med Rehabil. 2012;91(9): 814-820; doi: 10.1097/PHM.0b013e31825f13a3.

55. Day SJ, Altman DG. Blinding in clinical trials and other studies. BMJ. 2000;321(7259):504; doi: 10.1136/bmj. 321.7259 .504 